\title{
Assessment of racial differences in the incidence of thrombocytopenia induced by trastuzumab emtansine: a systematic review and meta-analysis
}

\author{
Jingyi Zhang ${ }^{1 \wedge}$, Yaning Yang ${ }^{1}$, Ru Chen ${ }^{2}$, Shanshan Chen ${ }^{1}$, Jiayu Wang ${ }^{1}$, Yang Luo ${ }^{1}$, Fei Ma $^{1}$, Binghe Xu$^{1}$, \\ Ying Fan $^{1}$
}

${ }^{1}$ Department of Medical Oncology, National Cancer Center/National Clinical Research Center for Cancer/Cancer Hospital, Chinese Academy of Medical Sciences and Peking Union Medical College, Beijing, China; ${ }^{2}$ National Central Cancer Registry, National Cancer Center/National Clinical Research Center for Cancer/Cancer Hospital, Chinese Academy of Medical Sciences and Peking Union Medical College, Beijing, China

Contributions: (I) Conception and design: Y Fan, J Zhang; (II) Administrative support: Y Fan, B Xu, F Ma; (III) Provision of study materials or patients: J Zhang, S Chen, J Wang, Y Luo; (IV) Collection and assembly of data: J Zhang, Y Yang, R Chen; (V) Data analysis and interpretation: J Zhang, Y Yang, R Chen; (VI) Manuscript writing: All authors; (VII) Final approval of manuscript: All authors.

Correspondence to: Prof. Dr. Ying Fan. Department of Medical Oncology, National Cancer Center/National Clinical Research Center for Cancer/ Cancer Hospital, Chinese Academy of Medical Sciences and Peking Union Medical College, No. 17 Panjiayuan Nanli, Chaoyang District, Beijing 100021, China. Email: fanyingfy@medmail.com.cn.

\begin{abstract}
Background: Trastuzumab emtansine (T-DM1) has been proved to have value and efficacy in the treatment of advanced metastatic cancer, including in the adjuvant setting. However, there is increasing concern about T-DM1-induced thrombocytopenia (TCP), which shows racial differences in incidence. This meta-analysis aimed to evaluate differences in the incidence of T-DM1-related TCP between Asian and non-Asian patients by combining accessible information from all single-agent T-DM1 clinical trials published to date.

Methods: We conducted systematic searches of the PubMed, Embase, and the Cochrane Library databases to identify relevant clinical studies of T-DM1 that reported on safety, including the incidence of TCP, which were published between January 1980 and March 2020. Two reviewers were responsible for the screening and extraction of data. The pooled-effect estimate calculated with a fixed-effects or random-effects model was represented as incidence with $95 \%$ confidence intervals (CIs).

Results: A total of 29 studies involving 6,188 patients were included. The incidence of all-grade TCP in Asian patients and non-Asian patients was 0.39 (95\% CI: 0.11-0.67) and 0.29 (95\% CI: 0.23-0.35), respectively. The incidence of TCP of grade 3 or higher in Asians was 0.20 (95\% CI: 0.10-0.29), compared with 0.02 (95\% CI: 0.01-0.03) in non-Asians. Gastrointestinal cancer type and a T-DM1 treatment dose of $2.4 \mathrm{mg} / \mathrm{kg}$ Q3W were related to grade 3 or higher TCP events.

Discussion: Asian patients have a higher risk of developing TCP after receiving T-DM1 than non-Asian patients. Clinicians should be aware of the importance of careful observation of platelet count in patients receiving T-DM1 therapy.
\end{abstract}

Keywords: Trastuzumab emtansine (T-DM1); thrombocytopenia (TCP); decreased platelet count; Asian

Submitted Apr 08, 2021. Accepted for publication Jul 15, 2021.

doi: $10.21037 / \mathrm{atm}-21-2763$

View this article at: https://dx.doi.org/10.21037/atm-21-2763

$\wedge$ ORCID: 0000-0003-4134-5649. 


\section{Introduction}

Overexpression of human epidermal growth factor receptor 2 (HER2) occurs in approximately $20 \%$ to $25 \%$ of breast cancer (BC) cases. The absence of anti-HER2 therapy is probably related to aggressive tumor growth and poor survival outcome of patients (1). Trastuzumab is the first monoclonal antibody to be developed against HER2. Research has shown that trastuzumab can benefit patients with metastatic HER2positive (HER2+) BC, improving their time to progression (TTP) and overall survival (OS) (2). However, the problem of drug resistance, which occurs in most advanced cases, has also been a challenge for researchers.

Lapatinib plus capecitabine has been evidenced to prolong the TTP, and has therefore been approved by the United States Food and Drug Administration (US FDA) as a treatment for patients with HER2 + metastatic BC (MBC) pretreated with trastuzumab (3). However, in a phase III randomized trial, only $29 \%$ of patients obtained a clinical benefit, and disease progression occurred in about $50 \%$ of the cases after median 6.2 months of therapy (4), suggesting a continuous requirement for the discovery of effective drugs aimed at HER2-positive disease.

Trastuzumab emtansine (T-DM1) is considered to be one of the most important antibody-drug conjugates in the BC field. T-DM1 combines the HER2-targeted antineoplastic effects of trastuzumab with the cytotoxicity of the microtubule inhibitor DM1 (a derivative of maytansine). A steady thioether linker MCC [(N-maleimidomethyl) cyclohexane-1-carboxylate] is responsible for the conjugation of the antibody and cytotoxic agent $(5,6)$. Upon binding to the extracellular domain of HER2, T-DM1 gains entry to the tumor cell through endocytosis mediated by receptors, and DM1 separates from the connectome via proteolysis, resulting in microtubule instability, cell cycle arrest, and apoptosis induction $(5,7)$. In addition to its role as a cytotoxic drug, T-DM1 maintains trastuzumab activity through the inhibition of signal transduction directed by HER2, giving rise to an antibody-dependent cell-mediated cytotoxicity effect (6). So far, phase 1 and phase 2 studies of T-DM1 have reported the results, and the conclusions of several large-scale randomized controlled phase 3 trials have demonstrated the significant clinical efficacy and safety of this drug for patients with HER2-positive MBC $(8,9)$.

With an incidence rate of $32 \%$, thrombocytopenia (TCP) is one of the most common adverse events (AEs) induced by T-DM1. And 12\% approximately of the patients had the highest grade of TCP (grade 3 and above) (10). Severe
TCP inevitably leads to dosage reduction, and in some cases, treatment termination; thus, it is a large obstacle to obtaining improved survival. TDM1-induced TCP is most likely mediated mainly via inhibition of megakaryocytic differentiation, with T-DM1 having no direct influence on platelet function (11). According to some studies, race is an important risk factor for TCP of grade 3 or higher $(12,13)$; however, the underpinning mechanism has not been clearly explained. There were several previous meta-analyses reporting the value and efficacy of T-DM1, however, these studies focused on all adverse events especially the common adverse events related to T-DM1, as well as survival outcome of the included patients. TCP is a common and potentially serious adverse event associated with T-DM1 use. Interestingly, we found that there likely to be racial variations between Asians and non-Asians in the risk of TCP. Race was shown to discriminate patient subgroups with significantly different risks of the occurrence of grade 3 TCP after T-DM1 initiation. We hence aimed at the racial differences in the incidence of TCP, in order to provide convinced evidence to support the clinical strategy of T-DM1 in different ethnicity. This meta-analysis aimed to provide credible evidence of differences in the incidence of T-DM1-induced TCP between Asian and non-Asian patients, which would be crucial to informing the rational use of T-DM1 for patients of different races. We present the following article in accordance with the PRISMA reporting checklist (available at https://dx.doi.org/10.21037/ atm-21-2763).

\section{Methods}

\section{Search strategy and selection criteria}

Systematic literature searches were undertaken to retrieve all available studies published between January1980 and March 2020, which reported AEs related T-DM1 treatment. The search process was conducted in the PubMed, Embase, and the Cochrane Library databases using the keywords "ado-trastuzumab emtansine", "ado-trastuzumab emtansine", "trastuzumab emtansine", "trastuzumab-DM1", "T-DM1", "TDM-1", and "Kadcyla". The search strategy took advantage of the availability of medical subject heading $(\mathrm{MeSH})$ terms and Emtree, using Boole's logical operators. The references of relevant studies and reviews were also reviewed for other potentially suitable studies. Eligible studies were selected on the basis of the following criteria: (I) oncotherapy study; (II) participants received T-DM1 as a 
single-agent treatment; (III) safety data on T-DM1-related AEs, including TCP, were reported; and (IV) English language. Articles published online ahead of print were also included. Conference abstracts, cell line and animal studies, and studies lacking patient information were deemed ineligible for inclusion. When several articles focused on the same cohort were identified, we included the article with the newest and/or most comprehensive AE data.

\section{Data extraction}

Literature retrieval, screening of the studies, and data extraction were independently carried out by 2 reviewers (JY.Z. and YN.Y.). Disagreements in opinion were decided by consensus, with the help of another reviewer (Y.F). The following characteristics were extracted from the included studies including: first author's name; country; publication year; study type; study phase; cancer type; mean age of participants; race of participants; T-DM1 dose density; dosing schedule; number of participants; and all AEs (grade $\geq 3$ ) and TCP events (all grades and grade $\geq 3$ ).

\section{Quality appraisal}

Two researchers used the Cochrane risk bias assessment tool to independently assess quality and bias of randomized controlled trials (RCT) in seven domains: random sequence generation; allocation concealment; blinding of participants and personnel; blinding of outcome assessment; incomplete outcome data; selective reporting and other sources of bias. Additionally, methodological index for non-randomized studies (MINORS) was used to assess the quality and bias of non-randomized clinical trials. The MINORS criteria contain eight items for non-comparative studies: a clearly stated aim; inclusion of consecutive patients; prospective collection of data; endpoints appropriate to the aim of the study; unbiased assessment of the study endpoint; follow-up period appropriate to the aim of the study; loss to follow-up less than 5\%; and prospective calculation of the study size. And the Joanna Briggs Institute (JBI) was used to assess the quality and risk bias of the observational studies. If the opinion of the two parties was not consistent, a third party's opinion was sought and discussed and resolved.

\section{Statistical analysis}

The R software 3.6.1, package 'meta' (metaprop and forest functions) was used for our data analysis. Fixed-effects and random-effects models were applied to assess the incidence of AEs, including TCP, associated with T-DM1 treatment in Asian, non-Asian, and mixed-race groups. Models were selected depending on the level of heterogeneity among the studies, as assessed using the $\mathrm{Q}$ statistic. $\mathrm{P}<0.10$ was taken as the significance level. The $\mathrm{I}^{2}$ statistic was also calculated to measure heterogeneity. $\mathrm{I}^{2}>50 \%$ was considered to be an indicator of high heterogeneity. In cases of statistical heterogeneity, a random-effects model was employed; otherwise, a fixed-effects model was used. $\mathrm{P} \leq 0.05$ was considered statistically significant. Forest plots were drawn and presented individual studies as horizontal solid lines with their confidence intervals (CIs), and the weight of the studies in the pooled estimate as proportional to the surface of the gray square. Using forest plots, we plotted the incidences of all-grade TCP, grade $\geq 3$ TCP events, and grade $\geq 3$ AEs, together with their $95 \%$ CIs, in the each cancer type and dose regimen. To detect publication bias, we carried out funnel plots and demonstrated probably publication bias existed.

\section{Results}

\section{Eligible studies and their characteristics}

The literature search yielded 3,787 publications. After careful screening against the eligibility criteria, a total of 29 studies involving 6,188 patients were finally enrolled in the metaanalysis (Figure 1; Table 1). The included studies consisted of 22 interventional trials and 7 observational studies. The proportion of Asians was totally 6.9\% ( $n=426)$, while the non-Asian and mixed-race groups accounted for $15.3 \%$ $(\mathrm{n}=949)$ and $77.8 \%(\mathrm{n}=4,813)$ respectively in this metaanalysis. The included studies focused on T-DM1 treatment for BC $(n=23)$, lung cancer $(n=3)$, gastrointestinal cancer $(n=2)$, and any other solid tumor $(n=1)$. The doses of T-DM1 used in the studies were $1.8 \mathrm{mg} / \mathrm{kg}$ every 3 weeks $(\mathrm{Q} 3 \mathrm{~W})$ $(\mathrm{n}=1), 2.4 \mathrm{mg} / \mathrm{kg} \mathrm{Q} 3 \mathrm{~W}(\mathrm{n}=1), 2.4 \mathrm{mg} / \mathrm{kg}$ every week $(\mathrm{QW})$ $(\mathrm{n}=2)$, and $3.6 \mathrm{mg} / \mathrm{kg}$ Q3W ( $=27)$. Two phase 1 clinical trials reported dose escalation, but they did not report the number of patients for each dose schedule; for this reason, we had to assign them into the mixed-dose regimen group. In their study plans, Thuss-Patience et al. and Shitara et al. performed two different T-DM1 dose regimens.

\section{Results of quality assessment}

Studies with various designs were included in this meta- 


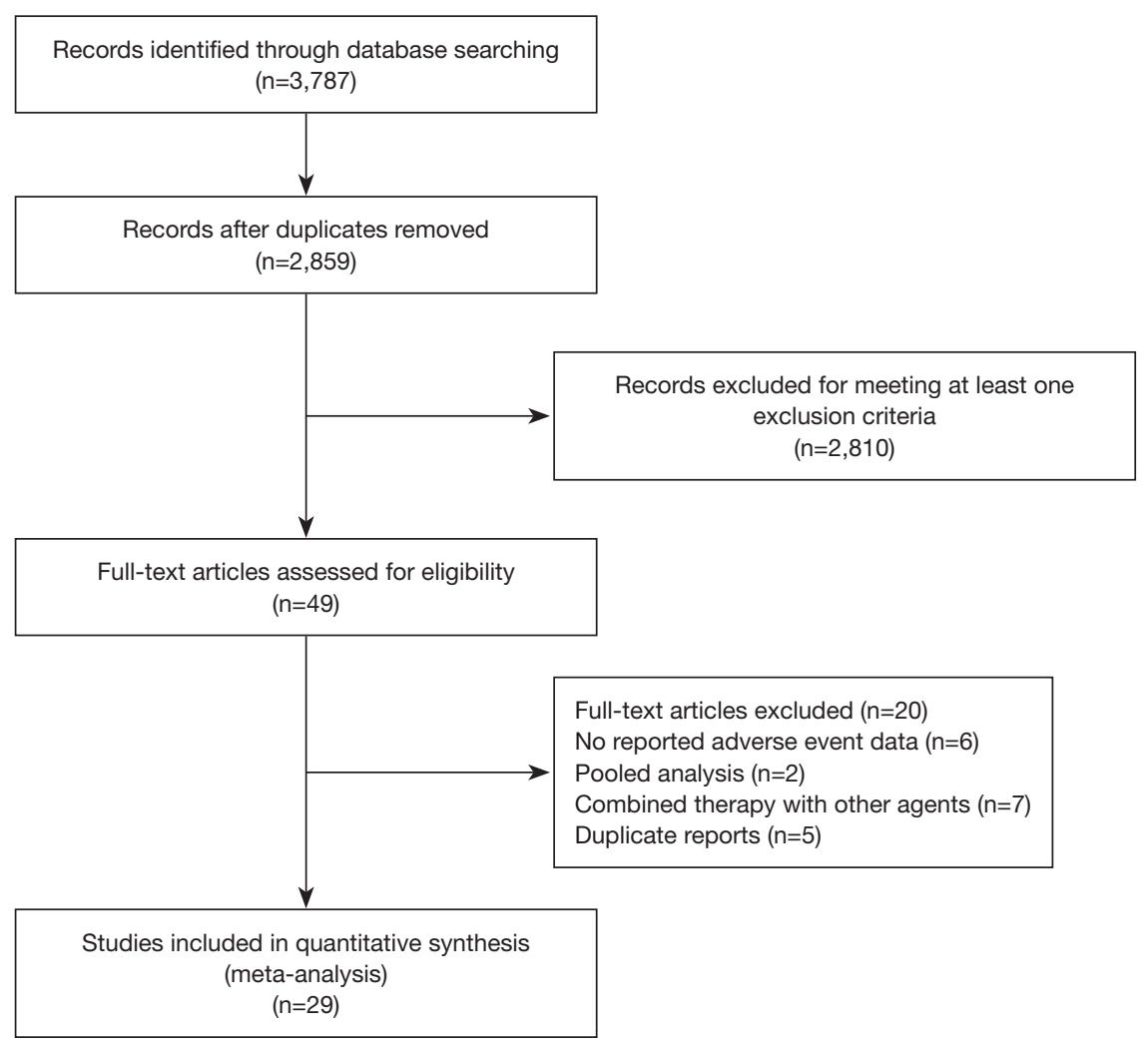

Figure 1 Flow diagram of the study selection process.

analysis and assessed accordingly with the appropriate scale or tool. The Cochrane risk bias assessment tool was used to assess the RCTs (Figure S1), while the MINORS scale was used for non-RCTs (Table S1) and JBI checklist was used for observational studies (Table S2). Overall, all included studies were appropriate for synthesis based on the criteria. In conclusion, all studies were deemed fit to be included in the meta-analysis.

\section{Incidence of TCP by race}

For all-grade TCP events related to T-DM1 treatment, 6,114 patients from 27 studies were divided into the Asian, non-Asian, and mixed-race groups for meta-analysis using the random-effects model. The 4 studies in the Asian group reported all-grade TCP events in 200 patients. The 11 studies in the mixed-race group reported all-grade TCP events in 888 patients. The 12 studies in the non-Asian group reported all-grade TCP events in 238 patients. The incidences of all-grade TCP events in the Asian, mixedrace, and non-Asian groups were $0.39 \%$ (95\% CI: $0.11-$ 0.67 ), $0.23 \%$ (95\% CI: $0.17-0.29$ ), and $0.29 \%$ (95\% CI:

\subsection{3-0.35) (Figure 2).}

The number of TCP events of grade $\geq 3$ was reported in 28 studies involving 5989 patients. The 6 studies in the Asian group reported grade $\geq 3$ TCP events in 101 patients, and synthetic results through meta-analysis using the random-effects model revealed an incidence of $0.20 \%$ (95\% CI: $0.10-0.29)$. The 11 studies in the mixed-race group reported grade $\geq 3$ TCP events in 284 patients who received T-DM1 monotherapy, and the incidence of grade $\geq 3$ TCP events was $0.07 \%$ (95\% CI: $0.05-0.10)$, as shown by random-effects model analysis. The 11 studies in the nonAsian group, which included 830 patients, reported 28 cases of grade $\geq 3$ TCP, translating to an incidence of $0.02 \%$ ( $95 \%$ CI: 0.01-0.03) according to fixed-effects model analysis (Figure 3).

Further analysis of the incidence of grade $\geq 3$ AEs related to T-DM1 monotherapy was carried out. A total of 25 studies involving 5,847 patients treated with T-DM1 reported 189, 1,819, and 112 cases of grade $\geq 3$ AEs in the Asian, mixed-race, and non-Asian groups, respectively; the incidence was 0.46 (95\% CI: $0.37-0.54), 0.41$ (95\% CI: $0.35-0.48)$, and 0.19 (95\% CI: 0.11-0.26), respectively 


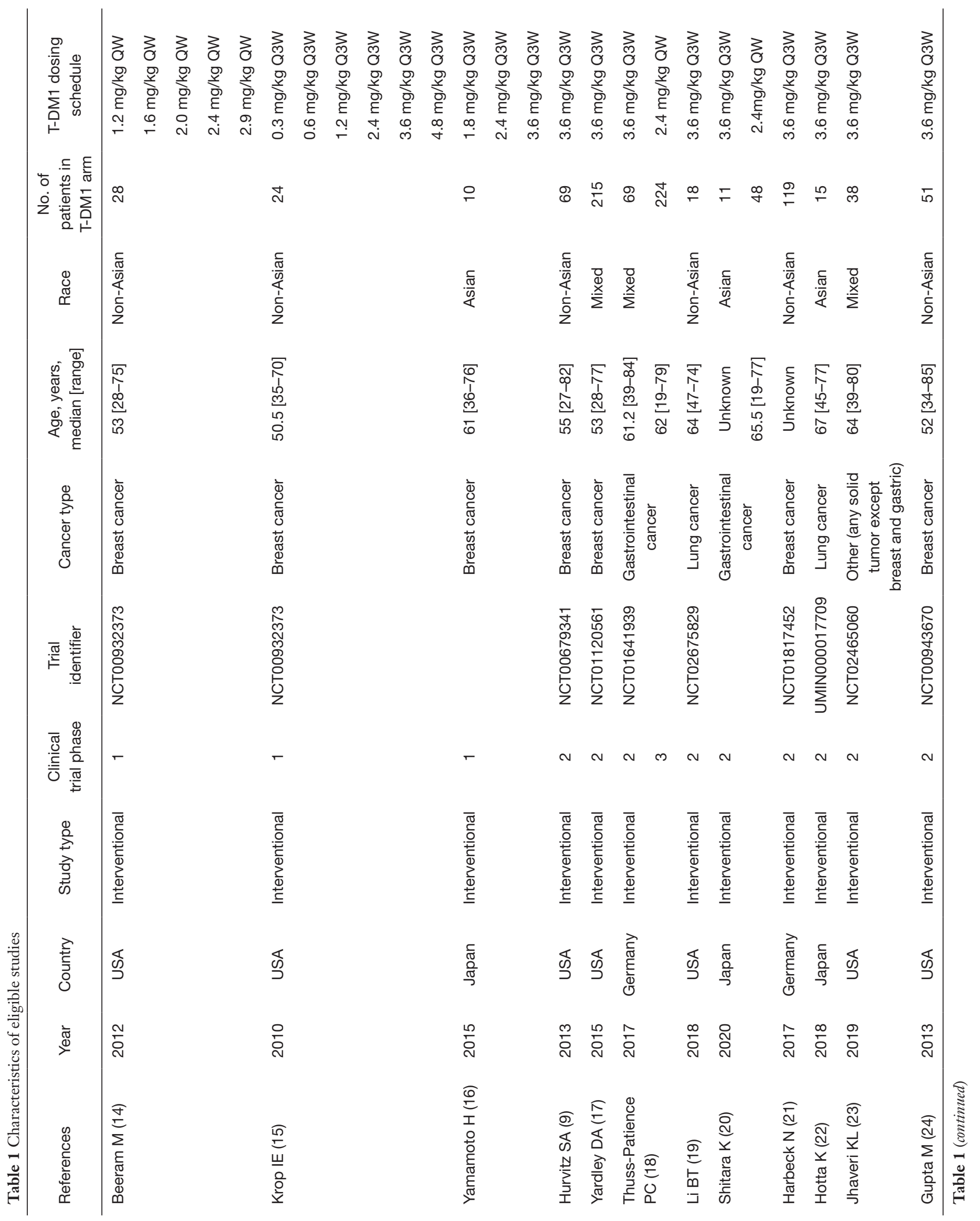




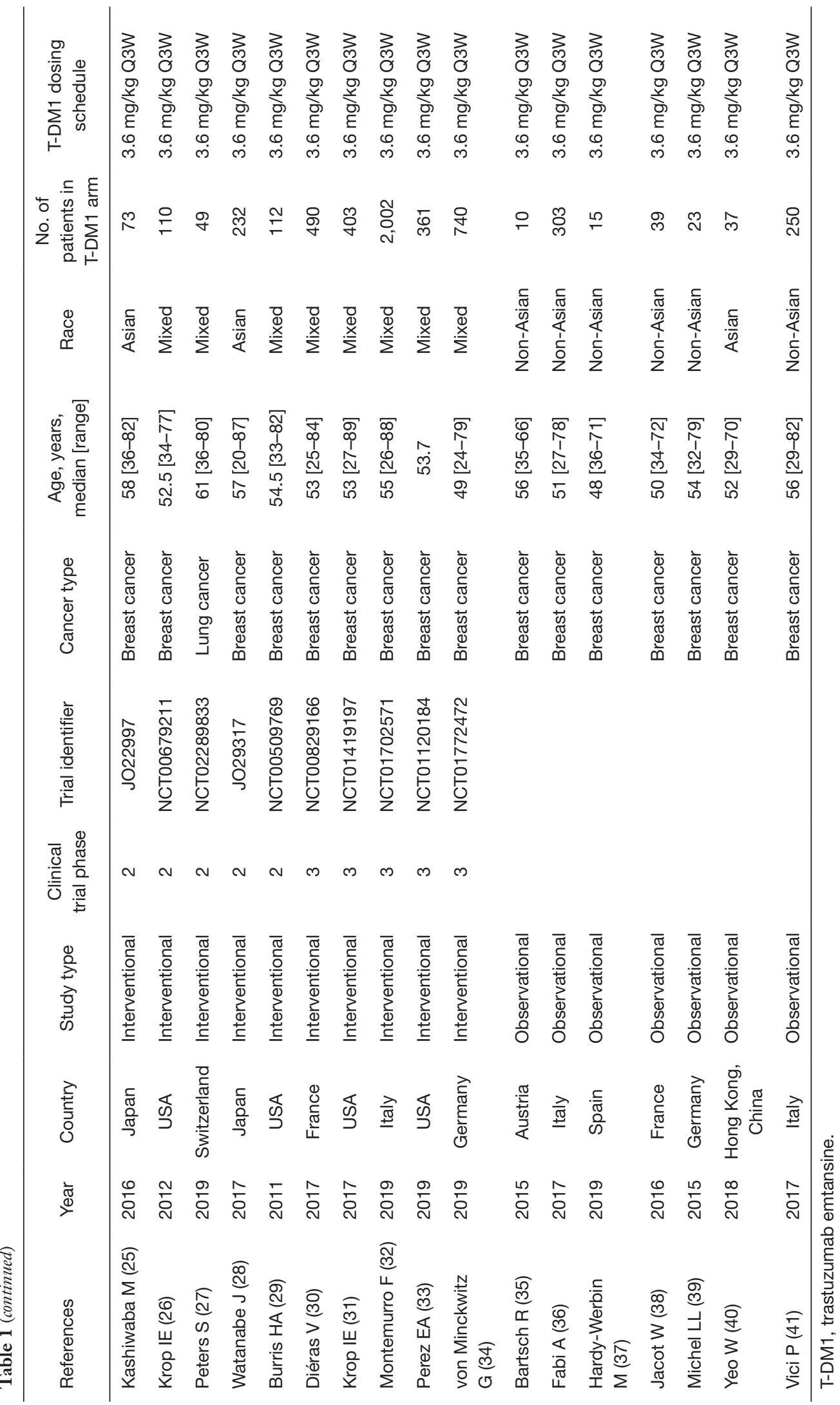




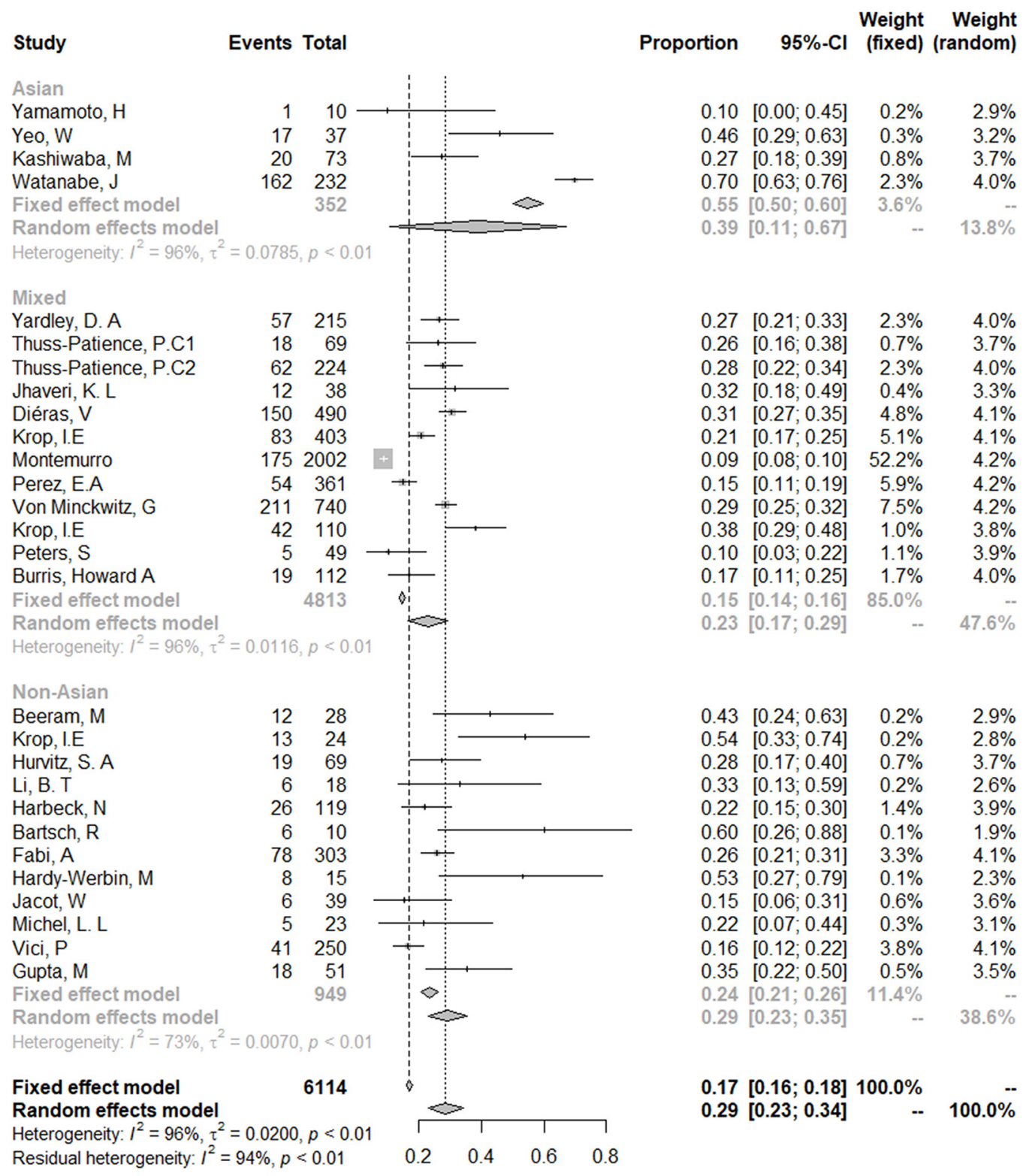

Figure 2 Incidence of the all-grade thrombocytopenia events related to T-DM1 by race. T-DM1, trastuzumab emtansine.

(Figure 4).

\section{Subgroup analysis of the incidence of TCP}

We next analyzed the incidence of all-grade TCP events, grade $\geq 3$ TCP events, and grade $\geq 3$ AEs according to the cancer type. The results showed that the incidence of allgrade TCP was high in the other solid tumors subgroup (except for BC and gastric cancer), and that gastrointestinal cancer was associated with a higher incidence of grade $\geq 3$ TCP events and grade $\geq 3$ AEs compared with other cancer types (Figure 5).

Additionally, subgroup analysis of dose regimens was also performed, which included $1.8 \mathrm{mg} / \mathrm{kg}$ Q3W, $2.4 \mathrm{mg} / \mathrm{kg}$ Q3W, $2.4 \mathrm{mg} / \mathrm{kg}$ QW, $3.6 \mathrm{mg} / \mathrm{kg}$ Q3W, and mixed-dose subgroups (no exact numbers of patients receiving different dose regimen). The subgroup analysis results showed that the dose of $2.4 \mathrm{mg} / \mathrm{kg}$ Q3W was associated with the high 


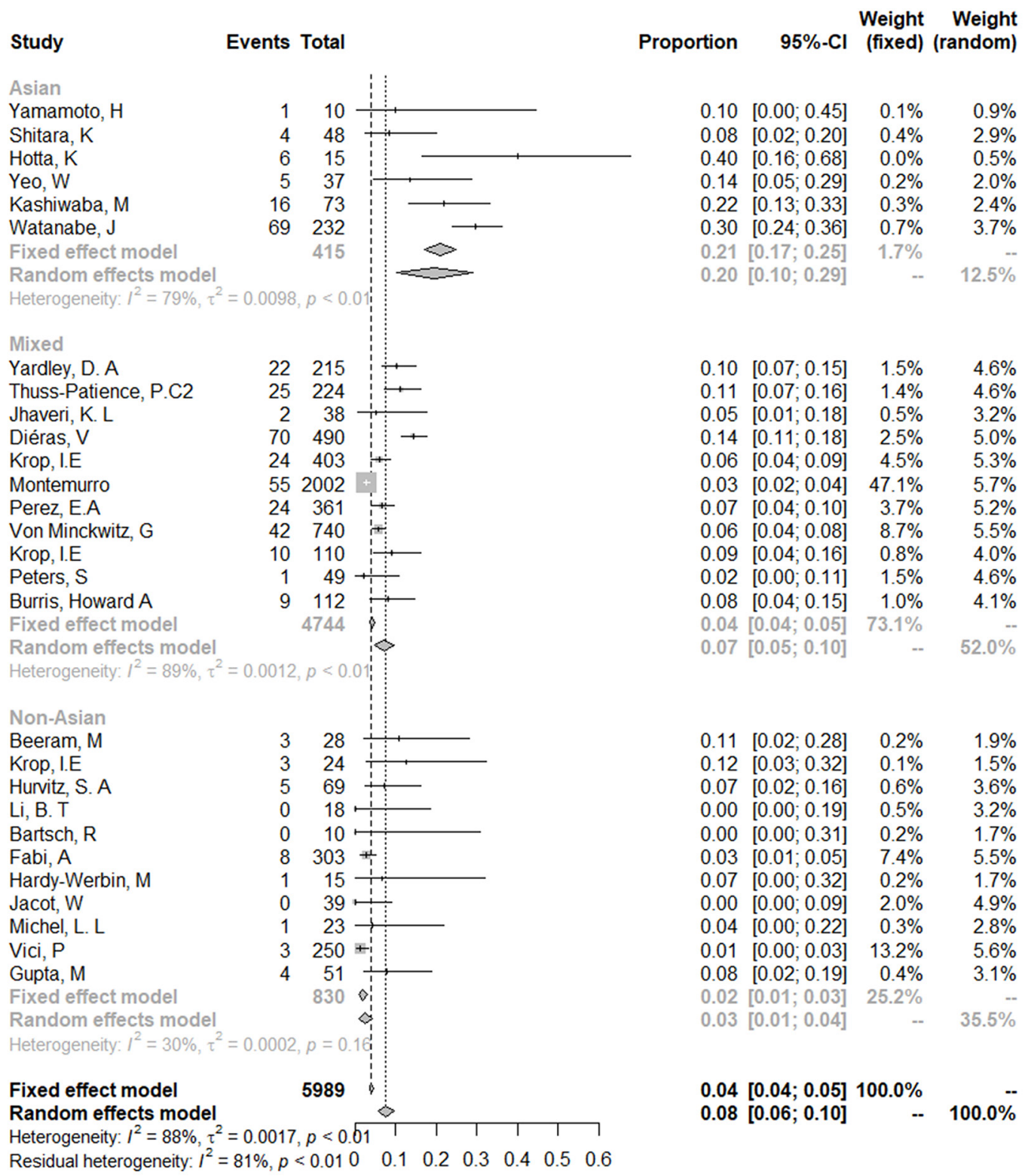

Figure 3 Incidence of grade $\geq 3$ thrombocytopenia events related to T-DM1 by race. T-DM1, trastuzumab emtansine.

incidence of grade $\geq 3$ TCP and grade $\geq 3$ AEs (Figure 6).

\section{Publication bias}

As shown in Figure S2, visual inspections of the funnel plots suggested an incidence dissymmetry distribution for allgrade TCP, while grade $\geq 3$ TCP probably was with low publication bias.

\section{Discussion}

In this meta-analysis involving 6,188 patients treated with T-DM1, a higher incidence of TCP was found in Asian patients than in non-Asian patients. Additionally, compared with non-Asian patients, more Asian patients experienced TCP events of grade $\geq 3$ after receiving T-DM1 therapy. Similarly, grade $\geq 3$ AEs occurred more in Asian patients 


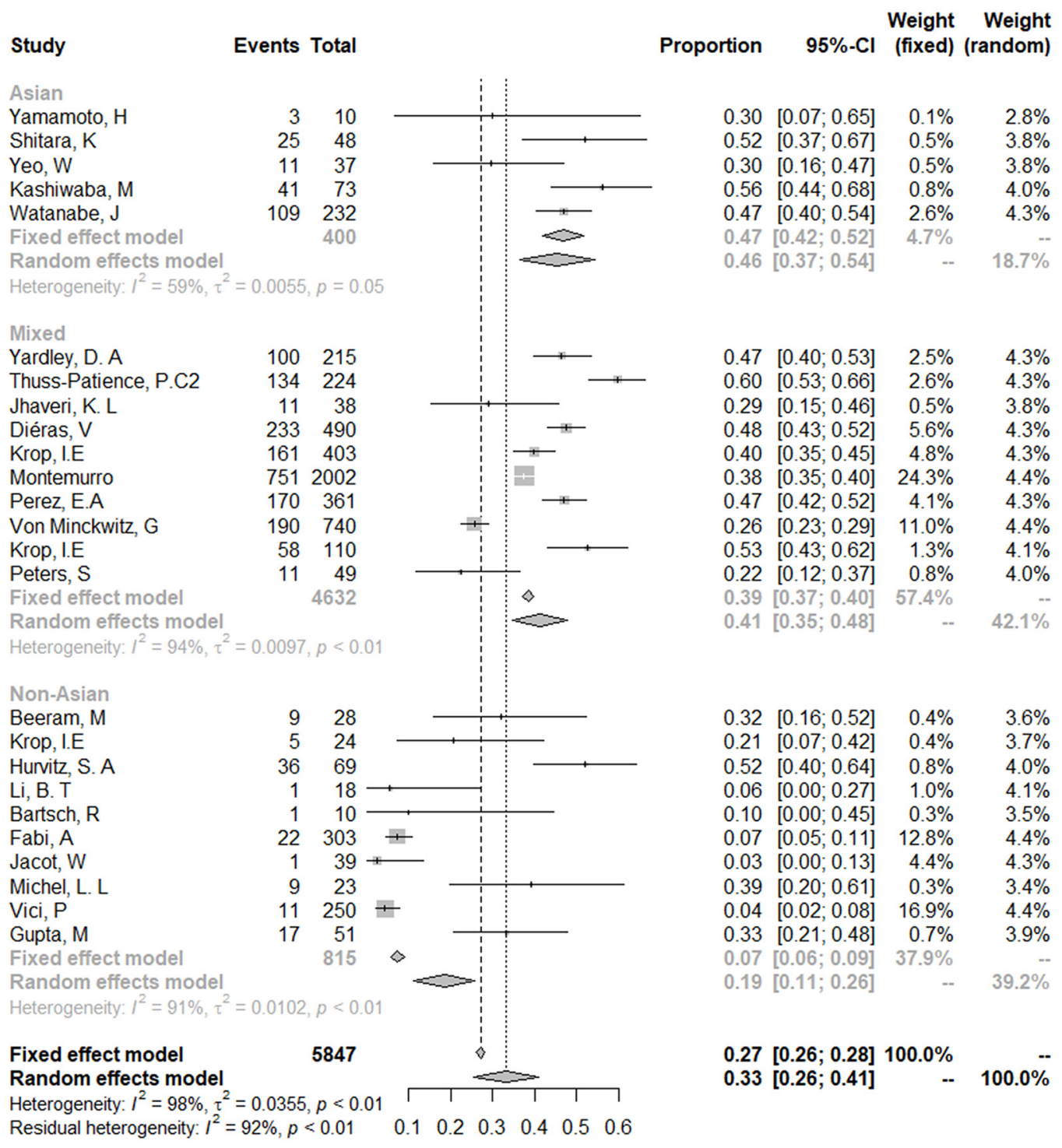

Figure 4 Incidence of grade $\geq 3$ adverse events related to T-DM1 by race. T-DM1, trastuzumab emtansine.

treated with T-DM1 than in non-Asian patients. This is the first meta-analysis to examine the safety profile of T-DM1 according to race, and the results indicate a significant difference in the incidence of T-DM1-induced TCP between Asian and non-Asian patients.

Consistently, the results from several large international clinical trials involving T-DM1 have suggested that race is a significant covariate for the risk of TCP, with Asian patients appearing to carry a higher risk than their nonAsian counterparts (13). T-DM1 was approved based on the significant results of the EMILIA trial, which compared the survival outcomes of patients with $\mathrm{BC}$ who received lapatinib plus capecitabine and those who received T-DM1 therapy (8). 358 HER2-positive, unresectable, locally advanced or MBC patients who were pretreated with trastuzumab and taxane, randomly entered into T-DM1 group, of which 94 Asian patients met the inclusion criteria. The data on the safety profile of T-DM1 showed that the most commonly occurring grade $\geq 3 \mathrm{AE}$ was TCP (12.9\%), and any-grade TCP occurred in $28.0 \%$ of cases in the T-DM1 group. Interestingly, the proportion of patients who experienced TCP events of any grade following T-DM1 
A

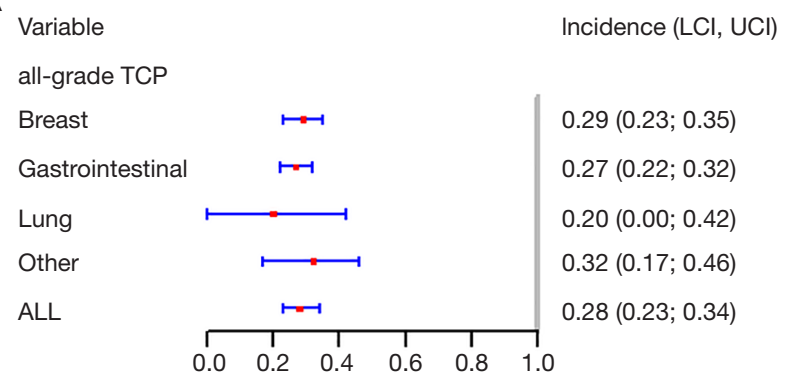

C

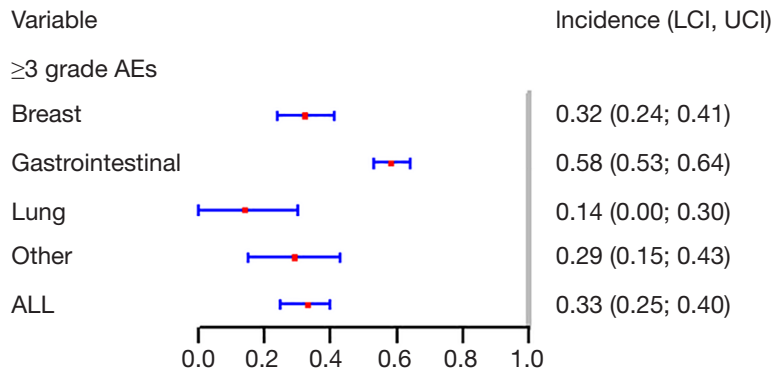

B

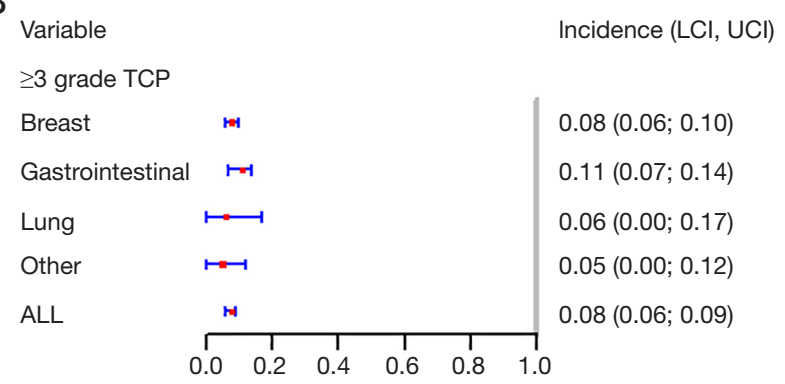

Figure 5 Incidence of thrombocytopenia and grade $\geq 3$ adverse events related to T-DM1 by cancer type. (A) Incidence of all-grade TCP related to T-DM1 by cancer type. (B) Incidence of grade 3 or higher TCP related to T-DM1 by cancer type. (C) Incidence of grade 3 or higher adverse events related to T-DM1 by cancer type. T-DM1, trastuzumab emtansine; TCP, thrombocytopenia; AEs, adverse events.

therapy was much higher among the Asian patient population (exceeding $45 \%$ ) than among the overall patient population. However, these AEs in the trials did not interrupt the treatment, and the majority of those patients were able to continue T-DM1 therapy after dose modification. Another multicenter, randomized, phase III trial, the KATHERINE trial, enrolled 1,486 patients with HER2+ early-stage BC with residual invasive disease after neoadjuvant therapy with a trastuzumab-containing regimen, including 101 Chinese participants (35). All patients accepted random assignment to the T-DM1 or trastuzumab arm. The safety analysis included 740 individuals who received T-DM1 therapy, 51 of whom were Chinese. According to the safety overview, TCP was found to be the most common T-DM1-induced grade $\geq 3 \mathrm{AE}$, affecting $6 \%$ of all patients and $22 \%$ of Chinese patients. Regarding all-grade TCP in the T-DM1 group, the incidence among Chinese patients was higher than that among the entire cohort (53\% vs. 29\%). Moreover, TCP was the most common reason for discontinuation of T-DM1 treatment. One Chinese patient in the T-DM1 arm who experienced TCP died from intracranial hemorrhage. Both of these clinical trials discovered a higher incidence of T-DM1induced TCP in Asian patients than non-Asian patients.
In our meta-analysis focusing on the safety profile of T-DM1, we also analyzed the mixed-race population which comprised patients of several races. The results showed that the incidence of all-grade TCP in Asian patients was higher than that in the mixed-race group (39\% vs. $23 \%$ ), with Asian patients also more likely than patients in the mixed-race group to experience TCP events of grade $\geq 3(20 \%$ vs. $7 \%)$. An integrated safety analysis of T-DM1 involving 884 patients from 6 studies who were treated with single-agent T-DM1 was conducted (12). The results revealed that $32.2 \%$ of T-DM1-treated patients experienced an TCP event of any grade, with $11.9 \%$ developing TCP of grade $\geq 3$. Asians who received T-DM1 exhibited a higher incidence of grade $\geq 3$ TCP than nonAsians (44.4\% vs. $10.6 \%$ ). Results of the pooled analysis were consistent with those of our meta-analysis, although we included more original clinical studies involving a larger number of patients.

As a common AE encountered during the course of T-DM1 treatment, TCP primarily results in drug dosage reduction, but it can even lead to withdrawal in some cases. However, the mechanism of TCP induced by T-DM1 has yet to be unearthed. High-content live-cell imaging assay 

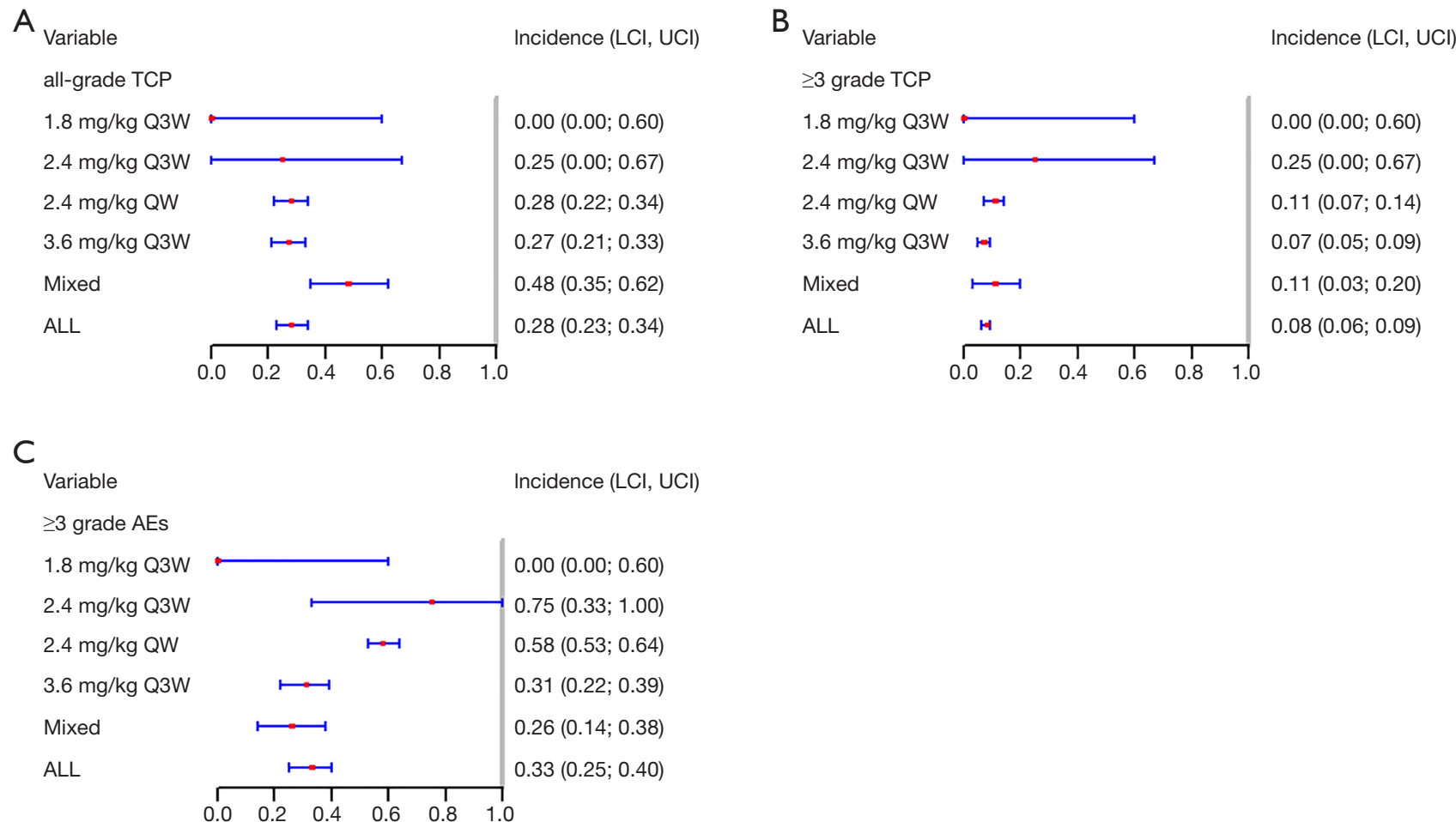

Figure 6 Incidence of thrombocytopenia and grade $\geq 3$ adverse events related to T-DM1 by treatment dose. (A) Incidence of all-grade TCP related to T-DM1 by treatment dose. (B) Incidence of grade 3 or higher TCP related to T-DM1 by treatment dose. (C) Incidence of grade 3 or higher adverse events related to T-DM1 by treatment dose. T-DM1, trastuzumab emtansine; TCP, thrombocytopenia; AEs, adverse events.

was ever used to establish mechanism of T-DM1-mediated inhibition of platelet production and found that T-DM1 is taken up by mouse megakaryocytes, inhibits megakaryocyte differentiation, and disrupts proplatelet formation by inducing abnormal tubulin organization and suppressing microtubule dynamic instability. More evidences are needed to reveal pathways by which T-DM1 affect megakaryocyte differentiation and proplatelet production (11). Actually, the occurrence of TCP has been infrequent with other HER2 monoclonal antibodies, even when combined with docetaxel and/or paclitaxel (42). T-DM1 was found to primarily inhibit megakaryocyte production from human hematopoietic stem cells rather than directly affect mature megakaryocytes. This effect was observed with T-DM1 but not with other HER2 monoclonal antibody, suggesting that the reductions in megakaryocyte and platelet production are independent of the antibody that DM1 is conjugated to, and mediated by DM1 (43).

A racial sensitivity assessment of T-DM1 found that Asians were possibly at a higher risk of developing TCP than non-Asians, which could not be explained by similar T-DM1 exposure observed between the two ethnic groups, but was probably connected with race-related differences in baseline platelet counts. However, the other integrated analysis held the opposite view that this ethnic difference of TCP induced by T-DM1 was independent of baseline platelet levels (12). An exploratory analysis presented a possible mechanism of the higher incidence of TCP among Asian patients, and considered that specific Fc polymorphisms identified more frequently in Asians are likely to have an effect on T-DM1-mediated TCP (44). Moreover, Osborne et al. described racial differences between Asian patients and non-Asian patients in the Fc $\gamma$ RIIa allotype (45). Previous population pharmacokinetics of T-DM1 found that the covariate of race was not statistically significant, suggesting that no further dose adjustment based on race was necessary (46). There was no apparent ethnic difference in the pharmacokinetics of T-DM1, suggesting that T-DM1 is unlikely to be sensitive to ethnic factors with respect to pharmacokinetics. And the 
pharmacokinetics of T-DM1 are unlikely to be affected by ethnic differences in HER2 expression. So far, no decent and convincing explanations of the main mechanisms were offered. Therefore, it was worthwhile to perform more trials in order to reveal the potential mechanisms of racial differences in incidence of TCP induced by T-DM1 (13). However, further pharmacodynamics analyses are needed to fully ascertain the reason why Asians carry a higher risk of TCP when receiving T-DM1 therapy. Further, the valuable clinical prediction model can also play a key role in quantifying the personalized risks of TCP after the initiation of T-DM1 therapy (47).

Some potential limitations still exist in our meta-analysis. Firstly, some race-related data were not available for some clinical trials, which might have led to bias. Besides, our meta-analysis was based on not only interventional study but also observational research, which might have negatively affected the quality assessment. However, with the safety data from ongoing clinical trials updated constantly, more sufficient evidence will become available in future.

As the first example of a successfully developed antibodydrug conjugate, T-DM1 entails the benefits of targeted delivery of chemotherapy and minimization of systemic toxicity. However, TCP still requires careful patient monitoring in order to make appropriate and timely dose adjustments (48). According to our analysis, the majority of TCP events were grade 1 or 2 , and those of grade $\geq 3$ did not rise to baseline across the whole cohort; in most cases, with appropriate dose modifications, platelet counts recovered to a level that permitted the continuation of therapy (12). Identifying patients who are more likely to experience TCP as early as possible is critical in order to give these patients preventive therapy to reduce side-effects during treatment. Clinicians also need to be vigilant and inform patients that if serious symptoms such as bleeding occur outside the hospital, they should return for thorough examination and treatments, such as platelet transfusion. If necessary, hematology specialist treatments may be required.

In conclusion, T-DM1 undoubtedly has significant efficacy, and its AEs are generally tolerable and controllable. Nevertheless, its safety profile in relation to TCP still raises clinical concerns. Asian patients have a higher risk of developing TCP than non-Asian patients. Monitoring platelet counts closely and regular follow-up are critically important. The safety profile of T-DM1 in Asian patients needs to be further explored in the future.

\section{Acknowledgments}

Funding: None.

\section{Footnote}

Reporting Checklist: The authors have completed the PRISMA reporting checklist. Available at https://dx.doi. org/10.21037/atm-21-2763

Conflicts of Interest: All authors have completed the ICMJE uniform disclosure form (available at https://dx.doi. org/10.21037/atm-21-2763). The authors have no conflicts of interest to declare.

Ethical Statement: The authors are accountable for all aspects of the work in ensuring that questions related to the accuracy or integrity of any part of the work are appropriately investigated and resolved.

Open Access Statement: This is an Open Access article distributed in accordance with the Creative Commons Attribution-NonCommercial-NoDerivs 4.0 International License (CC BY-NC-ND 4.0), which permits the noncommercial replication and distribution of the article with the strict proviso that no changes or edits are made and the original work is properly cited (including links to both the formal publication through the relevant DOI and the license). See: https://creativecommons.org/licenses/by-nc-nd/4.0/.

\section{References}

1. Lee HY, Shin IS, Rim CH. Benefits of adjuvant treatment including trastuzumab in HER2-positive pT1a-bN0M0 breast cancer: a systematic review and meta-analysis. Ann Transl Med 2020;8:187.

2. Slamon DJ, Leyland-Jones B, Shak S, et al. Use of chemotherapy plus a monoclonal antibody against HER2 for metastatic breast cancer that overexpresses HER2. N Engl J Med 2001;344:783-92.

3. Geyer CE, Forster J, Lindquist D, et al. Lapatinib plus capecitabine for HER2-positive advanced breast cancer. N Engl J Med 2006;355:2733-43.

4. Cameron D, Casey M, Press M, et al. A phase III randomized comparison of lapatinib plus capecitabine versus capecitabine alone in women with advanced breast cancer that has progressed on trastuzumab: updated 
efficacy and biomarker analyses. Breast Cancer Res Treat 2008;112:533-43.

5. Lewis Phillips GD, Li G, Dugger DL, et al. Targeting HER2-positive breast cancer with trastuzumab-DM1, an antibody-cytotoxic drug conjugate. Cancer Res 2008;68:9280-90.

6. Junttila TT, Li G, Parsons K, et al. Trastuzumab-DM1 (TDM1) retains all the mechanisms of action of trastuzumab and efficiently inhibits growth of lapatinib insensitive breast cancer. Breast Cancer Res Treat 2011;128:347-56.

7. Erickson HK, Park PU, Widdison WC, et al. Antibodymaytansinoid conjugates are activated in targeted cancer cells by lysosomal degradation and linker-dependent intracellular processing. Cancer Res 2006;66:4426-33.

8. Verma S, Miles D, Gianni L, et al. Trastuzumab emtansine for HER2-positive advanced breast cancer. N Engl J Med 2012;367:1783-91.

9. Hurvitz SA, Dirix L, Kocsis J, et al. Phase II randomized study of trastuzumab emtansine versus trastuzumab plus docetaxel in patients with human epidermal growth factor receptor 2-positive metastatic breast cancer. J Clin Oncol 2013;31:1157-63.

10. Shen K, Ma X, Zhu C, et al. Safety and Efficacy of Trastuzumab Emtansine in Advanced Human Epidermal Growth Factor Receptor 2-Positive Breast Cancer: a Meta-analysis. Sci Rep 2016;6:23262.

11. Thon JN, Devine MT, Jurak Begonja A, et al. Highcontent live-cell imaging assay used to establish mechanism of trastuzumab emtansine (T-DM1)--mediated inhibition of platelet production. Blood 2012;120:1975-84.

12. Diéras V, Harbeck N, Budd GT, et al. Trastuzumab emtansine in human epidermal growth factor receptor 2-positive metastatic breast cancer: an integrated safety analysis. J Clin Oncol 2014;32:2750-7.

13. Li C, Wang B, Lu D, et al. Ethnic sensitivity assessment of the antibody-drug conjugate trastuzumab emtansine ( $\mathrm{T}$ DM1) in patients with HER2-positive locally advanced or metastatic breast cancer. Cancer Chemother Pharmacol 2016;78:547-58.

14. Beeram M, Krop IE, Burris HA, et al. A phase 1 study of weekly dosing of trastuzumab emtansine (T-DM1) in patients with advanced human epidermal growth factor 2-positive breast cancer. Cancer 2012;118:5733-40.

15. Krop IE, Beeram M, Modi S, et al. Phase I study of trastuzumab-DM1, an HER2 antibody-drug conjugate, given every 3 weeks to patients with HER2-positive metastatic breast cancer. J Clin Oncol 2010;28:2698-704.

16. Yamamoto H, Ando M, Aogi K, et al. Phase I and pharmacokinetic study of trastuzumab emtansine in Japanese patients with HER2-positive metastatic breast cancer. Jpn J Clin Oncol 2015;45:12-8.

17. Yardley DA, Krop IE, LoRusso PM, et al. Trastuzumab Emtansine (T-DM1) in Patients With HER2-Positive Metastatic Breast Cancer Previously Treated With Chemotherapy and 2 or More HER2-Targeted Agents: Results From the T-PAS Expanded Access Study. Cancer J 2015;21:357-64.

18. Thuss-Patience PC, Shah MA, Ohtsu A, et al. Trastuzumab emtansine versus taxane use for previously treated HER2positive locally advanced or metastatic gastric or gastrooesophageal junction adenocarcinoma (GATSBY): an international randomised, open-label, adaptive, phase 2/3 study. Lancet Oncol 2017;18:640-53.

19. Li BT, Shen R, Buonocore D, et al. Ado-Trastuzumab Emtansine for Patients With HER2-Mutant Lung Cancers: Results From a Phase II Basket Trial. J Clin Oncol 2018;36:2532-7.

20. Shitara K, Honma Y, Omuro Y, et al. Efficacy of trastuzumab emtansine in Japanese patients with previously treated HER2-positive locally advanced or metastatic gastric or gastroesophageal junction adenocarcinoma: A subgroup analysis of the GATSBY study. Asia Pac J Clin Oncol 2020;16:5-13.

21. Harbeck N, Gluz O, Christgen M, et al. De-Escalation Strategies in Human Epidermal Growth Factor Receptor 2 (HER2)-Positive Early Breast Cancer (BC): Final Analysis of the West German Study Group Adjuvant Dynamic Marker-Adjusted Personalized Therapy Trial Optimizing Risk Assessment and Therapy Response Prediction in Early BC HER2- and Hormone ReceptorPositive Phase II Randomized Trial-Efficacy, Safety, and Predictive Markers for 12 Weeks of Neoadjuvant Trastuzumab Emtansine With or Without Endocrine Therapy (ET) Versus Trastuzumab Plus ET. J Clin Oncol 2017;35:3046-54.

22. Hotta K, Aoe K, Kozuki T, et al. A Phase II Study of Trastuzumab Emtansine in HER2-Positive Non-Small Cell Lung Cancer. J Thorac Oncol 2018;13:273-9.

23. Jhaveri KL, Wang XV, Makker V, et al. Ado-trastuzumab emtansine (T-DM1) in patients with HER2-amplified tumors excluding breast and gastric/gastroesophageal junction (GEJ) adenocarcinomas: results from the NCIMATCH trial (EAY131) subprotocol Q. Ann Oncol 2019;30:1821-30.

24. Gupta M, Wang B, Carrothers TJ, et al. Effects of Trastuzumab Emtansine (T-DM1) on QT Interval and 
Safety of Pertuzumab Plus T-DM1 in Patients With Previously Treated Human Epidermal Growth Factor Receptor 2-Positive Metastatic Breast Cancer. Clin Pharmacol Drug Dev 2013;2:11-24.

25. Kashiwaba M, Ito Y, Takao S, et al. A multicenter Phase II study evaluating the efficacy, safety and pharmacokinetics of trastuzumab emtansine in Japanese patients with heavily pretreated HER2-positive locally recurrent or metastatic breast cancer. Jpn J Clin Oncol 2016;46:407-14.

26. Krop IE, LoRusso P, Miller KD, et al. A phase II study of trastuzumab emtansine in patients with human epidermal growth factor receptor 2-positive metastatic breast cancer who were previously treated with trastuzumab, lapatinib, an anthracycline, a taxane, and capecitabine. J Clin Oncol 2012;30:3234-41.

27. Peters S, Stahel R, Bubendorf L, et al. Trastuzumab Emtansine (T-DM1) in Patients with Previously Treated HER2-Overexpressing Metastatic Non-Small Cell Lung Cancer: Efficacy, Safety, and Biomarkers. Clin Cancer Res 2019;25:64-72.

28. Watanabe J, Ito Y, Saeki T, et al. Safety evaluation of trastuzumab emtansine in Japanese patients with HER2positive advanced breast cancer. In Vivo 2017;31:493-500.

29. Burris HA, 3rd, Rugo HS, Vukelja SJ, et al. Phase II study of the antibody drug conjugate trastuzumab-DM1 for the treatment of human epidermal growth factor receptor 2 (HER2)-positive breast cancer after prior HER2-directed therapy. J Clin Oncol 2011;29:398-405.

30. Diéras V, Miles D, Verma S, et al. Trastuzumab emtansine versus capecitabine plus lapatinib in patients with previously treated HER2-positive advanced breast cancer (EMILIA): a descriptive analysis of final overall survival results from a randomised, open-label, phase 3 trial. Lancet Oncol 2017;18:732-42.

31. Krop IE, Kim SB, Martin AG, et al. Trastuzumab emtansine versus treatment of physician's choice in patients with previously treated HER2-positive metastatic breast cancer (TH3RESA): final overall survival results from a randomised open-label phase 3 trial. Lancet Oncol 2017;18:743-54.

32. Montemurro F, Ellis P, Anton A, et al. Safety of trastuzumab emtansine (T-DM1) in patients with HER2positive advanced breast cancer: Primary results from the KAMILLA study cohort 1. Eur J Cancer 2019;109:92-102.

33. Perez EA, Barrios C, Eiermann W, et al. Trastuzumab emtansine with or without pertuzumab versus trastuzumab with taxane for human epidermal growth factor receptor 2-positive advanced breast cancer: Final results from
MARIANNE. Cancer 2019;125:3974-84.

34. von Minckwitz G, Huang CS, Mano MS, et al. Trastuzumab Emtansine for Residual Invasive HER2Positive Breast Cancer. N Engl J Med 2019;380:617-28.

35. Bartsch R, Berghoff AS, Vogl U, et al. Activity of T-DM1 in Her2-positive breast cancer brain metastases. Clin Exp Metastasis 2015;32:729-37.

36. Fabi A, De Laurentiis M, Caruso M, et al. Efficacy and safety of T-DM1 in the 'common-practice' of HER2+ advanced breast cancer setting: A multicenter study. Oncotarget 2017;8:64481-9.

37. Hardy-Werbin M, Quiroga V, Cirauqui B, et al. Realworld data on T-DM1 efficacy - results of a single-center retrospective study of HER2-positive breast cancer patients. 2019;9:12760.

38. Jacot W, Pons E, Frenel JS, et al. Efficacy and safety of trastuzumab emtansine (T-DM1) in patients with HER2positive breast cancer with brain metastases. Breast Cancer Res Treat 2016;157:307-18.

39. Michel LL, Bermejo JL, Gondos A, et al. T-DM1 as a New Treatment Option for Patients with Metastatic HER2-positive Breast Cancer in Clinical Practice. Anticancer Res 2015;35:5085-90.

40. Yeo W, Luk MY, Soong IS, et al. Efficacy and tolerability of trastuzumab emtansine in advanced human epidermal growth factor receptor 2-positive breast cancer. Hong Kong Med J 2018;24:56-62.

41. Vici P, Pizzuti L, Michelotti A, et al. A retrospective multicentric observational study of trastuzumab emtansine in HER2 positive metastatic breast cancer: A real-world experience. Oncotarget 2017;8:56921-31.

42. Perez EA, Barrios C, Eiermann W, et al. Trastuzumab Emtansine With or Without Pertuzumab Versus Trastuzumab Plus Taxane for Human Epidermal Growth Factor Receptor 2-Positive, Advanced Breast Cancer: Primary Results From the Phase III MARIANNE Study. J Clin Oncol 2017;35:141-8.

43. Mahapatra K, Darbonne W, Bumbaca D, et al. T-DM1induced thrombocytopenia results from impaired platelet production in a HER2-independent manner. Mol Cancer Ther 2011;10:Abstract nr A135.

44. Uppal H, Doudement E, Mahapatra K, et al. Potential mechanisms for thrombocytopenia development with trastuzumab emtansine (T-DM1). Clin Cancer Res 2015;21:123-33.

45. Osborne JM, Chacko GW, Brandt JT, et al. Ethnic variation in frequency of an allelic polymorphism of human Fc gamma RIIA determined with allele 
specific oligonucleotide probes. J Immunol Methods 1994;173:207-17.

46. Lu D, Girish S, Gao Y, et al. Population pharmacokinetics of trastuzumab emtansine (T-DM1), a HER2-targeted antibody-drug conjugate, in patients with HER2positive metastatic breast cancer: clinical implications of the effect of covariates. Cancer Chemother Pharmacol 2014;74:399-410.

Cite this article as: Zhang J, Yang Y, Chen R, Chen S, Wang J, Luo Y, Ma F, Xu B, Fan Y. Assessment of racial differences in the incidence of thrombocytopenia induced by trastuzumab emtansine: a systematic review and meta-analysis. Ann Transl Med 2021;9(14):1139. doi: 10.21037/atm-21-2763
47. Modi ND, Sorich MJ, Rowland A, et al. Predicting Thrombocytopenia in Patients With Breast Cancer Treated With Ado-trastuzumab Emtansine. Clin Breast Cancer 2020;20:e220-8.

48. Mathew J, Perez EA. Trastuzumab emtansine in human epidermal growth factor receptor 2-positive breast cancer: a review. Curr Opin Oncol 2011;23:594-600. 


\section{Supplementary}
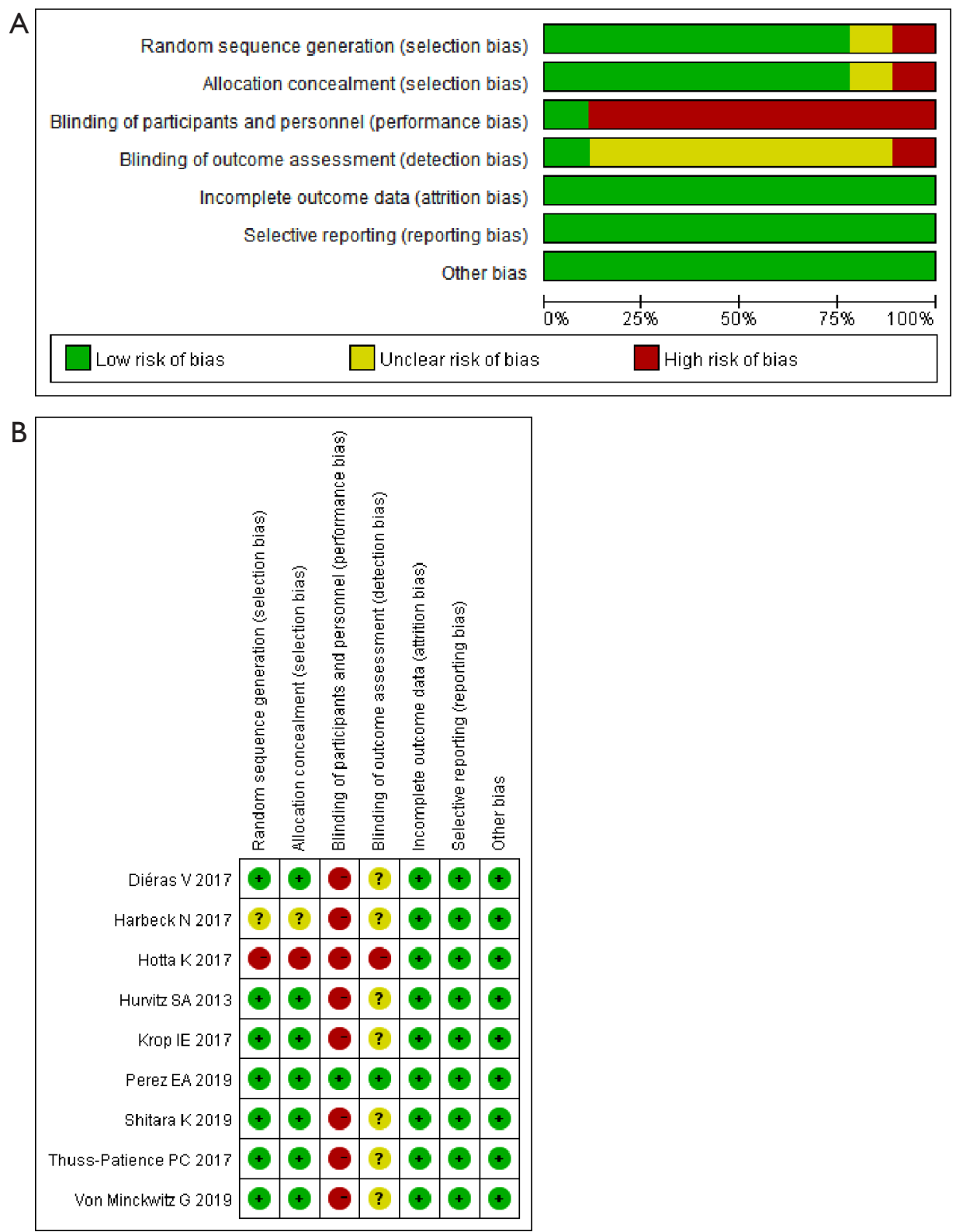

Figure S1 Quality assessment of randomized controlled trials by the Cochrane risk bias assessment tool. (A) Each risk of bias item presented as percentages across all included studies. (B) Each risk of bias item for each included study. 
Table S1 Methodological index for non-randomized studies

\begin{tabular}{|c|c|c|c|c|c|c|c|c|c|}
\hline & Clearly stated aim & $\begin{array}{c}\text { Inclusion of } \\
\text { consecutive patients }\end{array}$ & $\begin{array}{c}\text { Prospective } \\
\text { collection of data }\end{array}$ & $\begin{array}{l}\text { Endpoint appropriate to } \\
\text { the aim of the study }\end{array}$ & $\begin{array}{l}\text { Unbiased assessment of } \\
\text { the study endpoint }\end{array}$ & $\begin{array}{l}\text { Follow up period appropriate } \\
\text { to the aim of the study }\end{array}$ & $\begin{array}{l}\text { Loss of follow up } \\
\text { less than } 5 \%\end{array}$ & $\begin{array}{l}\text { Prospective calculation } \\
\text { of the study size }\end{array}$ & Total \\
\hline Beeram M 2012 & 2 & 0 & 2 & 2 & 0 & 2 & 2 & 0 & 10 \\
\hline Krop IE 2010 & 2 & 0 & 2 & 2 & 0 & 2 & 2 & 0 & 10 \\
\hline Yamamoto H 2015 & 2 & 0 & 0 & 2 & 0 & 2 & 2 & 0 & 8 \\
\hline Yardley DA 2015 & 2 & 2 & 0 & 2 & 2 & 2 & 2 & 1 & 13 \\
\hline Li BT 2018 & 2 & 1 & 2 & 2 & 2 & 2 & 2 & 1 & 14 \\
\hline Jhaveri KL 2019 & 2 & 1 & 1 & 2 & 1 & 2 & 2 & 1 & 12 \\
\hline Gupta M 2013 & 2 & 1 & 2 & 2 & 1 & 2 & 2 & 0 & 12 \\
\hline Kashiwaba M 2016 & 2 & 1 & 2 & 2 & 2 & 2 & 2 & 0 & 13 \\
\hline Krop IE 2012 & 2 & 1 & 2 & 2 & 2 & 2 & 2 & 1 & 14 \\
\hline Watanabe J 2017 & 2 & 2 & 1 & 1 & 2 & 2 & 2 & 0 & 12 \\
\hline Peters S 2018 & 2 & 1 & 2 & 2 & 1 & 2 & 2 & 1 & 13 \\
\hline Burris HA 2011 & 2 & 1 & 2 & 2 & 2 & 2 & 2 & 1 & 14 \\
\hline Montemurro F 2019 & 2 & 1 & 2 & 2 & 2 & 2 & 2 & 2 & 15 \\
\hline
\end{tabular}

Table S2 Joanna Briggs Institute Critical Appraisal tool for observational studies

\begin{tabular}{|c|c|c|c|c|c|c|c|c|c|}
\hline & $\begin{array}{l}\text { Were the criteria for } \\
\text { inclusion in the sample } \\
\text { clearly defined? }\end{array}$ & $\begin{array}{l}\text { Were the study } \\
\text { subjects and the settino } \\
\text { described in detail? }\end{array}$ & $\begin{array}{l}\text { Was the exposure } \\
\text { measured in a validc } \\
\text { and reliable way? }\end{array}$ & $\begin{array}{l}\text { Were objective, standard } \\
\text { criteria used for measurement } \\
\text { of the condition? }\end{array}$ & $\begin{array}{l}\text { Were confounding } \\
\text { factors identified? }\end{array}$ & $\begin{array}{l}\text { Were strategies to deal } \\
\text { with confounding factors } \\
\text { stated? }\end{array}$ & $\begin{array}{l}\text { Were the outcomes } \\
\text { measured in a valid and } \\
\text { reliable way? }\end{array}$ & $\begin{array}{c}\text { Was appropriate } \\
\text { statistical analysis } \\
\text { used? }\end{array}$ & Tota \\
\hline Bartsch R 2015 & 2 & 2 & 2 & 2 & 1 & 0 & 2 & 2 & 13 \\
\hline Fabi A 2017 & 2 & 2 & 1 & 2 & 0 & 0 & 2 & 2 & 11 \\
\hline Hardy-Werbin M 2019 & 1 & 0 & 1 & 2 & 0 & 0 & 2 & 2 & 8 \\
\hline Jacot W 2016 & 2 & 2 & 2 & 2 & 0 & 0 & 2 & 2 & 12 \\
\hline Michel LL 2015 & 0 & 0 & 2 & 1 & 0 & 0 & 2 & 2 & 7 \\
\hline Yeo W 2018 & 2 & 2 & 2 & 2 & 1 & 0 & 2 & 2 & 13 \\
\hline Vici P 2017 & 1 & 1 & 0 & 2 & 0 & 0 & 2 & 2 & 8 \\
\hline
\end{tabular}


A

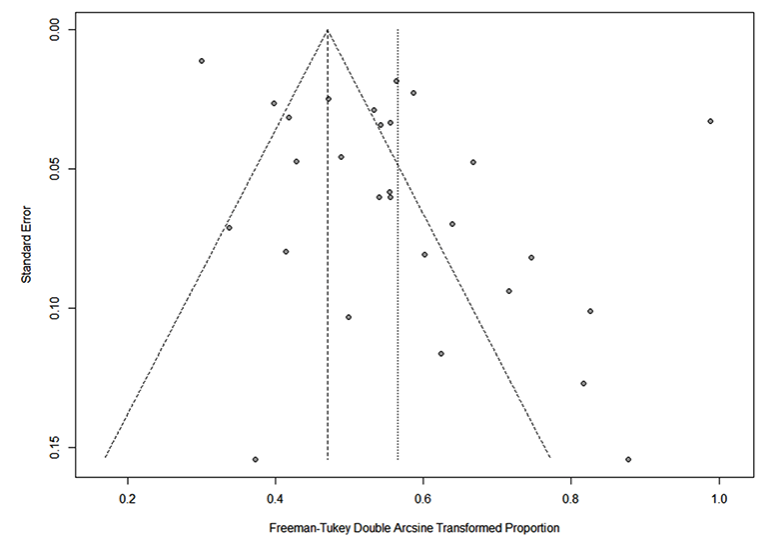

B

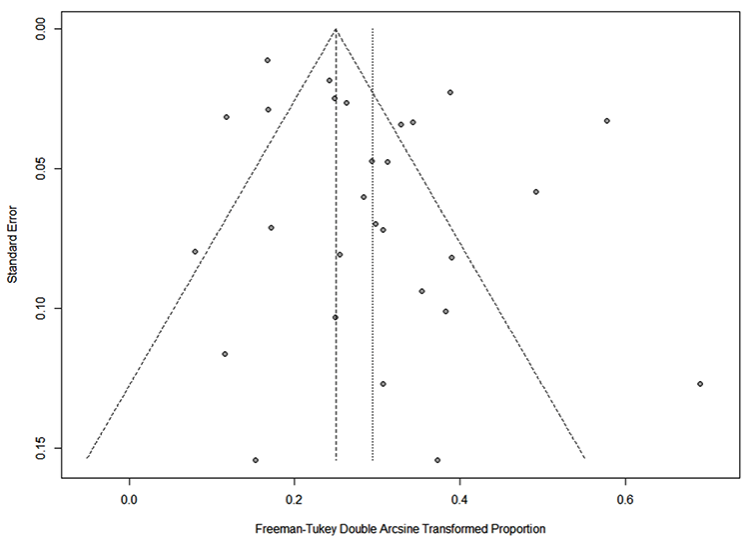

Figure S2 Funnel plots for the evaluation of the publication bias. (A) Publication bias in the incidence of all-grade thrombocytopenia. (B) Publication bias in the incidence of grade $\geq 3$ thrombocytopenia. 